\title{
Performance of Noncoherent Maximum-Likelihood Sequence Detection for Differential OFDM Systems With Diversity Reception
}

\author{
Ding-Bing Lin, Ping-Hung Chiang, and Hsueh-Jyh Li
}

\begin{abstract}
For the single-carrier M-ary differential phase-shift keying (MDPSK), the multiple-symbol differential detector, or the noncoherent maximum-likelihood sequence detector (NSD), and its three special cases, namely, the noncoherent one-shot detector, the linearly predictive decision-feedback (DF) detector, and the linearly predictive Viterbi receiver are reviewed based on a hierarchical interpretation. For the multicarrier transmission, the differential orthogonal frequency division multiplexing (OFDM) systems with diversity reception are discussed. It is well known that there are two types of differential OFDM systems, namely, the time domain differential OFDM (TD-OFDM) and the frequency domain differential OFDM (FD-OFDM). In this paper, the NSD and its special cases are incorporated to the differential OFDM systems. Furthermore, we provide a simple closed-form bit-error-rate (BER) expression for the differential OFDM systems utilizing the noncoherent one-shot detector with diversity reception in the time-varying multipath Rayleigh fading channels. Numerical results have revealed that, with multi-antenna diversity reception, the performance of the noncoherent one-shot detector is improved significantly. However, when only one or two receive antennas are available, the implementation of the linearly predictive DF detector or the linearly predictive Viterbi receiver is necessary for achieving better and satisfactory performance.
\end{abstract}

Index Terms-Decision-feedback differential detection, differential orthogonal frequency division multiplexing (OFDM), estimator-detector, noncoherent maximum-likelihood sequence detection (NSD), noncoherent one-shot detection, Viterbi algorithm.

\section{INTRODUCTION}

$\mathbf{F}$ OR the single-carrier M-ary differential phase-shift keying (MDPSK) [1], [2], Divsalar et al. [3] and Ho et al. [4] proposed the multiple-symbol differential detector, or the noncoherent maximum-likelihood (ML) sequence detector, to improve the conventional product detector [1], or the noncoherent one-shot detector. Since the noncoherent one-shot detector is built assuming that the channel is constant over two consecutive symbol durations, its bit-error-rate (BER) performance exhibits an error floor in the time-selective fading channel. It is proved theoretically that the noncoherent ML sequence detector (NSD) can lower this error floor significantly by detecting a sequence of symbols jointly [4]. Although the NSD is optimal, its complexity increases exponentially with the number of symbols

Manuscript received May 27, 2004; revised August 9, 2005. This work was supported by the National Science Council, Republic of China, under Grant NSC 93-2213-E027-035.

D.-B. Lin is with the Department of Electronic Engineering, National Taipei University of Technology, Taipei 106, Taiwan, R.O.C. (e-mail: dblin@ en.ntut.edu.tw).

P.-H. Chiang and H.-J. Li are with the Graduate Institute of Communication Engineering, National Taiwan University, Taipei 10617, Taiwan, R.O.C. (e-mail: d1942011@ee.ntu.edu.tw; hjli@ew.ee.ntu.edu.tw).

Digital Object Identifier 10.1109/TBC.2005.857604 being jointly detected. To reduce its complexity and obtain the satisfactory performance simultaneously, the linearly predictive decision-feedback (DF) detector [5] and the linearly predictive Viterbi receiver [6]-[8] were proposed. Generally, the metrics used by the above two detection schemes contain a linear predictor of the fading-plus-noise processes. Therefore, they have an attractive estimator-detector structure.

Orthogonal frequency-division multiplexing (OFDM) is an excellent technique to reduce the effect of frequency-selective fading by dividing the transmission bandwidth into many narrow-band subcarriers, each of which exhibits an approximately flat fading [9], [10]. For this multicarrier scheme, the differential modulation can be applied on each subcarrier, and the corresponding differential encoding and detection can be performed in either the time domain or frequency domain. According to the direction of the differential encoding and detection (see Fig. 1), the differential OFDM systems are classified into two categories: 1) the time domain differential OFDM (TD-OFDM) [10]-[15]; 2) the frequency domain differential OFDM (FD-OFDM) [10], [13]. The former has been standardized in the terrestrial digital audio broadcasting (DAB) system [16].

Nevertheless, only the noncoherent one-shot detector was considered in the above cited papers regarding the differential OFDM systems. In this paper, the NSD and its three special cases, namely, the noncoherent one-shot detector, linearly predictive DF detector, and linearly predictive Viterbi receiver are incorporated to the differential OFDM systems with diversity reception. Furthermore, we provide a simple closed-form bit-error-rate (BER) expression for the differential OFDM systems utilizing the noncoherent one-shot detector with diversity reception in the time-varying multipath Rayleigh fading channels.

The remaining of the paper is organized as follows. In Section II, a hierarchical interpretation of the NSD and its three special cases is presented. In Section III, the preliminaries of the differential OFDM systems with diversity reception are described. In Section IV, the receiver designs of the differential OFDM systems according to the NSD and its three special cases are illustrated. Also, a simple BER expression for the differential OFDM systems employing the noncoherent one-shot detector is given. Numerical results are shown in Section V, whereas the conclusions are drawn in Section VI.

\section{Noncoherent ML Sequence Detector}

In this section, the NSD with diversity reception is reviewed. Then, three special cases of the NSD are introduced, namely, the noncoherent one-shot detector, linearly predictive DF detector, and linearly predictive Viterbi receiver. At the end of this 


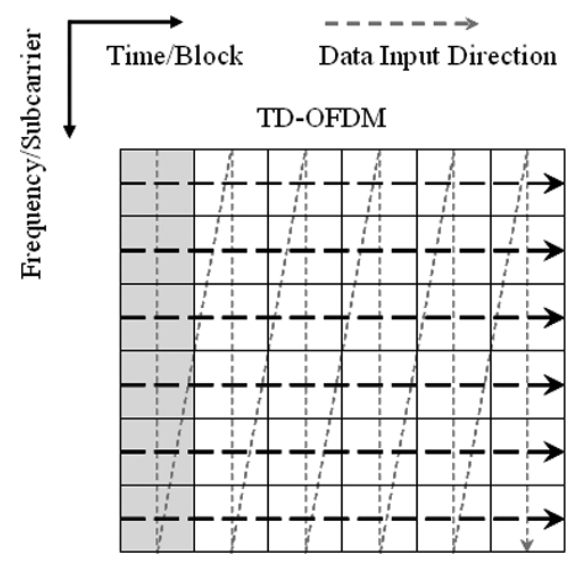

Fig. 1. Comparison between the TD- and FD-OFDM.

section, a hierarchical interpretation of the NSD and its special cases is also given.

For the single-carrier transmission, an $N_{T}$-symbol sequence is assumed to be transmitted and received over a singleinput-multiple-output (SIMO) time-varying flat Rayleigh fading channel. Let $v_{n}$ denote the $n$th information symbol, which is a $L$-PSK symbol, i.e., $v_{n} \in \Omega_{v}$ and $\Omega_{v}=\{\exp (j 2 \pi l / L)$, $l=0,1, \cdots, L-1\}$. Each information symbol is differentially encoded as follows [1], [2].

$$
x_{n}=v_{n} x_{n-1}, \quad x_{0}=1 \text {. }
$$

Obviously, the differentially encoded symbol, or channel symbol, $x_{n}$ still belongs to the $L$-ary constellation $\Omega_{v}$. Then, collecting the received signal from all $Q$ receive antennas gives

$$
\mathbf{r}_{n}=x_{n} \mathbf{h}_{n}+\mathbf{w}_{n},
$$

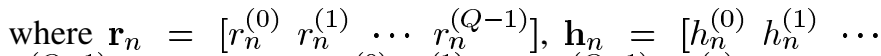

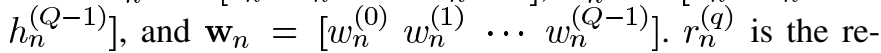
ceived signal from the $q$ th receive antenna in the $n$th symbol interval. $h_{n}^{(q)}$ is the path gain with mean zero, variance $\phi_{0} \triangleq 1$, and autocorrelation $\phi_{m} \triangleq \mathrm{E}\left[h_{n+m}^{(q)} h_{n}^{(q)^{*}}\right]$, assuming $Q$ identical and independent channels. $w_{n}^{(q)}$ is the AWGN with mean zero and variance $\sigma_{w}^{2}$. Suppose that the transmission begins at time $n=0$ and ends at time $n=N_{T}-1$. Stacking up the variables involved in the optimum block detection yields the signal model as

$$
\mathbf{R}=\mathbf{X H}+\mathbf{W}
$$

where $\mathbf{R}=\left[\mathbf{r}_{0}^{T} \mathbf{r}_{1}^{T} \cdots \mathbf{r}_{N_{T}-1}^{T}\right]^{T}, \mathbf{X}=\operatorname{diag}\left\{x_{0}, x_{1}, \cdots\right.$, $\left.\left.x_{N_{T}-1}\right\}, \quad \mathbf{H}={ }=\mathbf{h}_{0}^{T} \mathbf{h}_{1}^{T} \cdots \mathbf{h}_{N_{T}-1}^{T}\right]^{T}, \quad$ and $\mathbf{W}=\left[\begin{array}{llll}\mathbf{w}_{0}^{T} & \mathbf{w}_{1}^{T} & \cdots & \mathbf{w}_{N_{T}-1}^{T}\end{array}\right]^{T}$. From (1), it is clear that there exists a one-to-one correspondence between the $\left(N_{T}-1\right) \times 1$ information symbol vector $\mathbf{v}=\left[\begin{array}{lllll}v_{1} & v_{2} & \cdots & v_{N_{T}-1}\end{array}\right]^{T}$ and the $N_{T} \times 1$ channel symbol vector $\mathbf{x}=\left[\begin{array}{llll}x_{0} & x_{1} & \cdots & x_{N_{T}-1}\end{array}\right]^{T}$. From (3), it can be shown that the ML estimate of $\mathbf{v}$ is obtained through

$$
\hat{\mathbf{v}}=\underset{\mathbf{v}}{\arg \min }\left\{\operatorname{tr}\left(\mathbf{R}^{H} \mathbf{X} \boldsymbol{\Phi}^{-1} \mathbf{X}^{H} \mathbf{R}\right)\right\}
$$
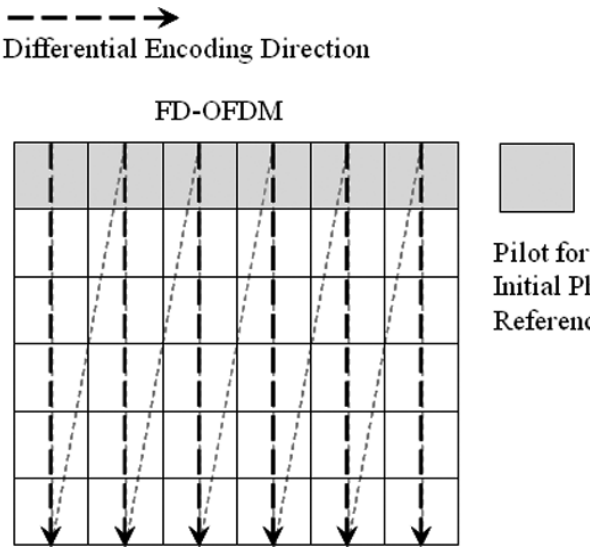

Pilot for

Initial Phase

Reference

which is the noncoherent sequence detector [4]. Here $\boldsymbol{\Phi}=\mathbf{C}_{\mathbf{h}}+\sigma_{w}^{2} \mathbf{I}_{N_{T}}$ and $\mathbf{C}_{\mathbf{h}}$ is the covariance matrix of

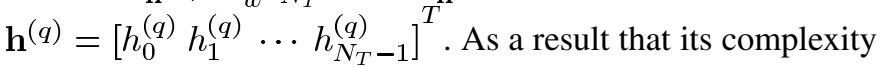
grows exponentially with $N_{T}$, the direct implementation of this detector is not recommended. In the following subsections, we introduce three special cases of this NSD, which can be carried out in practice.

\section{A. Linearly Predictive Viterbi Receiver}

The NSD given in (4) brings a linear-prediction interpretation of its structure. To avoid that its complexity increases exponentially with $N_{T}$, the minimization problem in (4) is solved by using the innovations-based approach [6], which is mathematically equivalent to the Cholesky factorization approach [7] [17,Ch. 3.7] as follows. Applying the Cholesky factorization to the $N_{T} \times N_{T}$ matrix $\boldsymbol{\Phi}$ produces

$$
\Phi=\mathbf{L} \Sigma \mathbf{L}^{H}
$$

where $\mathbf{L}$ is a $N_{T} \times N_{T}$ lower triangular matrix and $\boldsymbol{\Sigma}=\operatorname{diag}\left\{\varepsilon_{0}, \varepsilon_{1}, \cdots, \varepsilon_{N_{T}-1}\right\}$. The inverse of $\mathbf{L}$ is denoted as

$$
\mathbf{L}^{-1}=\left[\begin{array}{ccccc}
-p_{0}^{0} & 0 & 0 & \cdots & 0 \\
-p_{1}^{1} & -p_{0}^{1} & 0 & \cdots & 0 \\
-p_{2}^{2} & -p_{1}^{2} & -p_{0}^{2} & \cdots & 0 \\
\vdots & \vdots & \vdots & \ddots & \vdots \\
-p_{N_{T}-1}^{N_{T}-1} & -p_{N_{T}-2}^{N_{T}-1} & -p_{N_{T}-3}^{N_{T}-1} & \cdots & -p_{0}^{N_{T}-1}
\end{array}\right] \text { (6) }
$$

Note that the element $p_{0}^{n}=-1$ and $p_{k}^{n}$ is the $k$ th coefficient of the $n$th order one-step forward linear predictor for the fadingplus-noise process $\left\{h_{n}^{(q)}+x_{n}^{*} w_{n}^{(q)}\right\}$ and $\varepsilon_{n}$ is the corresponding mean square prediction error. Then, substituting (5) and (6) into (4) results in a linearly predictive sequence detector as

$$
\hat{\mathbf{v}}=\underset{\mathbf{v}}{\arg \min } \sum_{n=0}^{N_{T}-1} \frac{\left\|\mathbf{y}_{n}-\hat{\mathbf{y}}_{n}\right\|^{2}}{\varepsilon_{n}}
$$

where $\hat{\mathbf{y}}_{n}=\sum_{k=1}^{n} p_{k}^{n} x_{n-k}^{*} \mathbf{r}_{n-k}$ is the $n$th order prediction of the fading-plus-noise vector $\mathbf{y}_{n}=x_{n}^{*} \mathbf{r}_{n}=\mathbf{h}_{n}+x_{n}^{*} \mathbf{w}_{n}$. 
To further limit the complexity, the estimator-detector structure given in (7) is simplified to an approximate ML sequence detector, containing a $K$ th order linear predictor, as

$$
\begin{aligned}
\hat{\mathbf{v}} & =\frac{\underset{\mathbf{v}}{\arg \min } \sum_{n=0}^{N_{T}-1}\left\|x_{n}^{*} \mathbf{r}_{n}-\sum_{k=1}^{K} p_{k}^{K} x_{n-k}^{*} \mathbf{r}_{n-k}\right\|^{2}}{\varepsilon_{K}} \\
& =\underset{\mathbf{v}}{\arg \min } \sum_{n=0}^{N_{T}-1} \sum_{q=0}^{Q-1}\left|x_{n}^{*} r_{n}^{(q)}-\sum_{k=1}^{K} p_{k}^{K} x_{n-k}^{*} r_{n-k}^{(q)}\right|^{2},
\end{aligned}
$$

where $\varepsilon_{K}$, a constant not affecting the detection, is dropped. Based on (8), a trellis with $L^{K}$ states is defined as follows. Each state of the trellis in the $n$th symbol interval is represented as $\boldsymbol{\Gamma}_{n}=\left[\begin{array}{llll}x_{n-K} & x_{n-K+1} & \cdots & x_{n-1}\end{array}\right]$. There are $L$ transitions emerging from each state and terminating in $L$ different states. The branch metric associated with each transition is defined as

$$
\begin{aligned}
\Delta\left(\boldsymbol{\Gamma}_{n}, v_{n}\right) & =\left\|x_{n}^{*} \mathbf{r}_{n}-\sum_{k=1}^{K} p_{k}^{K} x_{n-k}^{*} \mathbf{r}_{n-k}\right\|^{2} \\
& =\sum_{q=0}^{Q-1}\left|x_{n}^{*} r_{n}^{(q)}-\sum_{k=1}^{K} p_{k}^{K} x_{n-k}^{*} r_{n-k}^{(q)}\right|^{2} .
\end{aligned}
$$

Then the Viterbi algorithm [18,Ch. 19] with a fixed decision delay $D$ can be applied to this trellis for solving the minimization problem in (8).

The algorithmic complexity of the above trellis-based sequence detector is proportional to the number of states $L^{K}$ which may be very large. Accordingly, the reduced-state sequence detection [8] can be incorporated to overcome the implementation complexity. A reduced state $\tilde{\boldsymbol{\Gamma}}_{n}$ is defined with the most recent $U$ channel symbols, namely, $\tilde{\boldsymbol{\Gamma}}_{n}=\left[\begin{array}{llll}x_{n-U} & x_{n-U+1} & \cdots & x_{n-1}\end{array}\right]$, and $\mathrm{U}<\mathrm{K}$. The calculation of the branch metric $\Delta\left(\tilde{\boldsymbol{\Gamma}}_{n}, v_{n}\right)$ involves $K+1$ observations and $K+1$ trial channel symbols. Thus, there are still $K-U$ channel symbols $x_{n-K}, x_{n-K+1}, \cdots, x_{n-U-1}$ unavailable in the state $\tilde{\boldsymbol{\Gamma}}_{n}$. However, one can extract these unavailable channel symbols from the survivor history according to the per-survivor processing (PSP) technique [8]. Thereupon, the branch metric for this reduced-state approach is written as

$$
\begin{aligned}
\Delta\left(\tilde{\boldsymbol{\Gamma}}_{n}, v_{n}\right)=\sum_{q=0}^{Q-1} \mid x_{n}^{*} r_{n}^{(q)} & -\sum_{k=1}^{U} p_{k}^{K} x_{n-k}^{*} r_{n-k}^{(q)} \\
& -\left.\sum_{k=U+1}^{K} p_{k}^{K} \tilde{x}_{n-k}^{*} r_{n-k}^{(q)}\right|^{2},
\end{aligned}
$$

where $\tilde{x}_{n-K}, \tilde{x}_{n-K+1}, \cdots, \tilde{x}_{n-U-1}$ are determined by the survivor entering the state $\tilde{\Gamma}_{n}$. Subsequently, this reduced-state trellis-based sequence detector [7] is named the Viterbi receiver (VR).

\section{B. Linearly Predictive Decision-Feedback Detector}

Here, we consider an extreme case of $U=0$ for the VR. In this case, only a single path is allowed to survive, then all the $K$ channel symbols required by the estimator are found from the history of this survivor and hence the sequence detector degenerates into a DF detector performing symbol-by-symbol detection as

$$
\begin{aligned}
\hat{v}_{n} & =\underset{v_{n}}{\arg \min }\left\|x_{n}^{*} \mathbf{r}_{n}-\sum_{k=1}^{K} p_{k}^{K} \hat{x}_{n-k}^{*} \mathbf{r}_{n-k}\right\|^{2} \\
& =\underset{v_{n}}{\arg \min } \sum_{q=0}^{Q-1}\left|x_{n}^{*} r_{n}^{(q)}-\sum_{k=1}^{K} p_{k}^{K} \hat{x}_{n-k}^{*} r_{n-k}^{(q)}\right|^{2},
\end{aligned}
$$

which is exactly the linearly predictive DF detector [5]. Subsequently, this detector is called the decision-feedback receiver (DR).

\section{Noncoherent One-Shot Detector}

Considering the DR with the prediction order $K=1,(11)$ is reduced to

$$
\begin{aligned}
& \hat{v}_{n}=\underset{v_{n}}{\arg \min } \sum_{q=0}^{Q-1}\left|x_{n}^{*} r_{n}^{(q)}-p_{1}^{1} \hat{x}_{n-1}^{*} r_{n-1}^{(q)}\right|^{2} \\
& =\underset{v_{n}}{\arg \max } \Re\left[\left(p_{1}^{1}\right)^{*} v_{n}^{*} \sum_{q=0}^{Q-1} r_{n}^{(q)} r_{n-1}^{(q)^{*}}\right] \\
& =\underset{v_{n}}{\arg \max } \Re\left(\phi_{1}^{*} v_{n}^{*} \sum_{q=0}^{Q-1} r_{n}^{(q)} r_{n-1}^{(q)^{*}}\right) \text {, }
\end{aligned}
$$

provided that $p_{1}^{1}=\phi_{1} /\left(\phi_{0}+\sigma_{w}^{2}\right)$. (12) is the noncoherent one-shot detector and is dubbed the conventional receiver (CR), subsequently.

Ignoring the fading correlation $\phi_{1}$ in (12) results in a suboptimum detector as

$$
\hat{v}_{n}=\underset{v_{n}}{\arg \max } \Re\left(v_{n}^{*} \sum_{q=0}^{Q-1} r_{n}^{(q)} r_{n-1}^{(q)^{*}}\right),
$$

which is the well-known product detector [1]. For this suboptimum detector, Kam derived a closed-form BER expression as follows [2].

$$
P_{b}=\left(\frac{1-\mu}{2}\right)^{Q} \sum_{q=0}^{Q-1}\left(\begin{array}{c}
Q-1+q \\
q
\end{array}\right)\left(\frac{1+\mu}{2}\right)^{q}
$$

where $\mu=\left|\phi_{1}\right| \Gamma /(1+\Gamma)$ for differential BPSK (i.e. $L=2$ and $\mu=\left\{2\left[(1+\Gamma) /\left(\left|\phi_{1}\right| \Gamma\right)\right]^{2}-1\right\}^{-1 / 2}$ for differential QPSK (i.e. $L=4$ ). $\Gamma=\phi_{0} / \sigma_{w}^{2}$ is the average signal-to-noise-ratio (SNR) per receiving branch. Obviously, (14) can be an upper bound for the BER of the CR given by (12).

\section{Hierarchy of the NSD and Its Special Cases}

Now we interpret the hierarchy of the NSD (4) and its three special cases, namely, the CR (12), DR (11), and VR (10). Firstly, the VR is a suboptimum version of the NSD; secondly, the DR is the one-survivor version of the VR; finally, the $\mathrm{CR}$ is the one-order-prediction version of the DR. Moreover, since the NSD and its special cases are inherently of the estimator-detector structure, their performances are dominated by the qualities of their estimates regarding the fading-plus-noise 


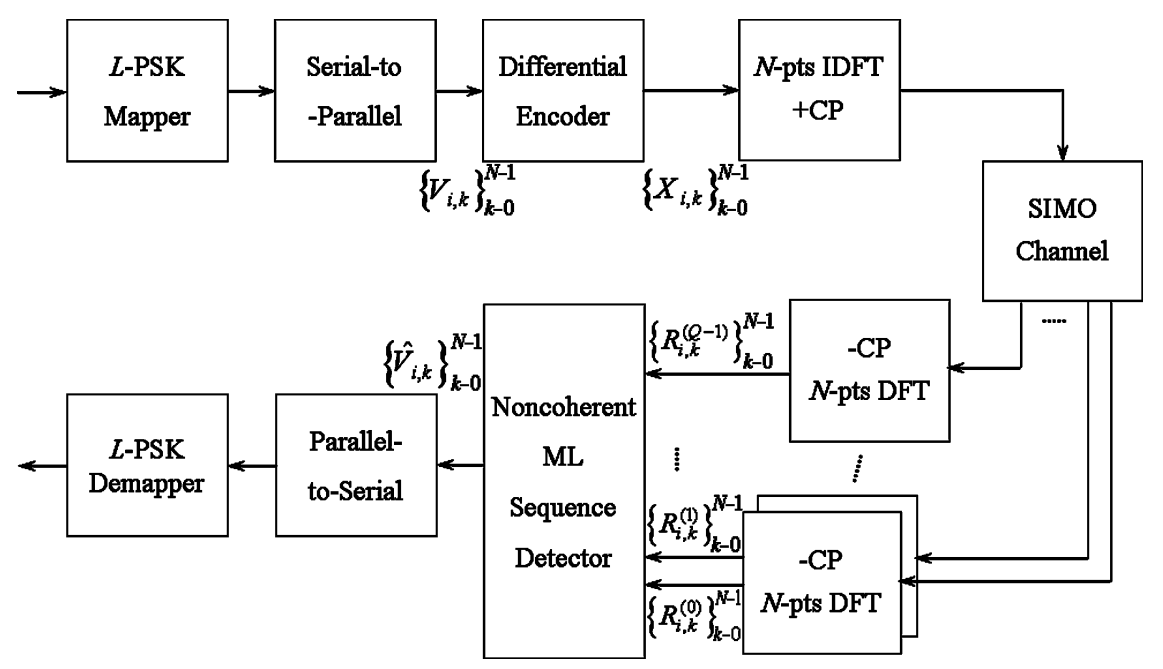

Fig. 2. Discrete-time baseband equivalent system model for the differential OFDM.

processes. More specifically, the VR employs the PSP technique so that each state in the trellis has its own survivor. Then the estimates contained in the branch metrics are different from state to state or from path to path. Indeed, the VR benefits from the path diversity and thus performs better than its one-survivor version, namely, the DR. Furthermore, with a larger prediction order and hence better estimates, the DR performs better than its one-order-prediction version, i.e. the CR.

\section{PRELIMINARIES FOR DifFERENTIAL OFDM Systems WITH DIVERSITY RECEPTION}

It is well known that there are two types of differential OFDM systems, namely, the TD- and FD-OFDM. The comparison is illustrated in Fig. 1. In this section, the system model and some statistical properties of the differential OFDM systems with diversity reception are provided. Then, the receiver designs will be discussed later in Section IV.

\section{A. System Model}

As shown in Fig. 2, we consider the DFT-based OFDM transmission over the SIMO WSSUS Rayleigh fading channel and assume sufficient cyclic prefix (CP) is inserted such that the inter-block-interference (IBI) is eliminated completely [9]. Let $V_{i, k}$ denote the $L$-PSK information symbol for the $k$ th subcarrier in the $i$ th OFDM block interval. Then, the channel symbol $X_{i, k}$ is produced via the differential encoding as [10], [13]

$$
X_{i, k}=\left\{\begin{array}{lll}
V_{i, k} X_{i-1, k}, & X_{0, k}=1 & (\text { TD-OFDM }) \\
V_{i, k} X_{i, k-1}, & X_{i, 0}=1 & \text { (FD-OFDM) }
\end{array}\right.
$$

Let $N, G$, and $M$ represent the number of subcarriers, guard samples, and taps, respectively. Then, the discrete-time baseband representation of the received signal from the $q$ th receive antenna is [9], [19]

$$
R_{i, k}^{(q)}=H_{i, k}^{(q)} X_{i, k}+W_{i, k}^{(q)}, \text { for } 0 \leq k \leq N-1,
$$

where $W_{i, k}^{(q)}=C_{i, k}^{(q)}+N_{i, k}^{(q)}$ is the equivalent AWGN [19] with mean zero and variance $\sigma_{W}^{2}=\sigma_{C}^{2}+N_{0}$. Here, $H_{i, k}^{(q)}, C_{i, k}^{(q)}$, and $N_{i, k}^{(q)}$ are the multiplicative distortion (MD), inter-carrier interference (ICI), and frequency domain noise, respectively.

\section{B. Statistical Properties}

For the ease of demonstrating the receiver designs later in Section IV, the statistical properties of the MD $H_{i, k}^{(q)}$, and ICI $C_{i, k}^{(q)}$ are clarified. Considering the exponential power delay profile [20] with the constraint $\sum_{m=0}^{M-1} \sigma_{m}^{2}=1$, the fading power of the $m$ th tap is given by

$$
\sigma_{m}^{2}=\frac{1-\lambda}{1-\lambda^{M}} \lambda^{m}
$$

where $\lambda=e^{-1 / d}$ and the delay control $d$ dominates the normalized root-mean-square (RMS) delay spread $\tau_{r m s} / T$. Here $T$ is the symbol duration (or the reciprocal of the system bandwidth).

1) Correlation of the Multiplicative Distortion: For the spatially independent and identical WSSUS Rayleigh fading channels with the classical Doppler spectrum, let $\rho_{H}^{(q)}(\Delta i, \Delta k) \triangleq$ $\rho_{H}(\Delta i, \Delta k)$, for $q=0,1, \cdots, Q-1$, where $\rho_{H}^{(q)}(\Delta i, \Delta k)$ is the correlation of $H_{i, k}^{(q)}$. Then, the correlation of the MD is given as [19]

$$
\begin{aligned}
\rho_{H}(\Delta i, \Delta k) \triangleq & \mathrm{E}\left[H_{i+\Delta i, k+\Delta k}^{(q)} H_{i, k}^{(q)^{*}}\right] \\
= & \left(\sum_{m=0}^{M-1} \sigma_{m}^{2} e^{-j \frac{2 \pi \Delta k m}{N}}\right)\left(\frac{1}{N^{2}} \sum_{l=-N+1}^{N-1}(N-|l|)\right. \\
& \left.\times J_{0}\left\{2 \pi f_{D} T[(N+G) \Delta i+l]\right\}\right),
\end{aligned}
$$

where $J_{0}(\cdot)$ is the zero-order Bessel function of the first kind and $f_{D}$ is the maximum Doppler frequency. Thereupon, the time correlation $\rho_{t} \triangleq \rho_{H}(1,0)$ can characterize the channel timeselectivity, while the frequency correlation $\rho_{f} \triangleq \rho_{H}(0,1)$ can represent the channel frequency-selectivity.

2) Variances of the Multiplicative Distortion and ICI: From [19], it is shown that the MD $H_{i, k}^{(q)}$ is of mean zero and hence 
variance $\sigma_{H}^{2}=\rho_{H}(0,0)$. In addition, a tight upper bound on the variance of the ICI $C_{i, k}^{(q)}$ is given by [21]

$$
\sigma_{C}^{2} \leq \frac{1}{24}\left(2 \pi f_{D} N T\right)^{2} E_{S}
$$

This approximation is quite accurate for $f_{D} N T<0.15$, even though it is derived assuming an infinite number of subcarriers.

\section{RECEIVER DESIGNS FOR DIFFERENTIAL OFDM SYSTEMS WITH DIVERSITY RECEPTION}

In this section, the NSD and its special cases, i.e., the CR, DR, and VR, introduced in Section II, are applied to the differential OFDM systems, namely, the TD- and FD-OFDM. The theoretical BER's for both systems employing the CR are also given.

\section{A. TD-OFDM}

For the TD-OFDM, since the differential encoding and detection are independent and simultaneous on all $N$ subcarriers (see Fig. 1), only the detection on the $k$ th subcarrier is illustrated subsequently. In light of (2) and (16), the $1 \times Q$ received signal vector for the $k$ th subcarrier in the $i$ th block interval from all $Q$ receive antennas is

$$
\mathbf{r}_{i, k}=X_{i, k} \mathbf{h}_{i, k}+\mathbf{w}_{i, k},
$$

where $\mathbf{r}_{i, k}=\left[R_{i, k}^{(0)} R_{i, k}^{(1)} \cdots R_{i, k}^{(Q-1)}\right], \mathbf{h}_{i, k}=\left[H_{i, k}^{(0)} H_{i, k}^{(1)} \cdots\right.$ $\left.H_{i, k}^{(Q-1)}\right]$, and $\mathbf{w}_{i, k}=\left[\begin{array}{llll}W_{i, k}^{(0)} & W_{i, k}^{(1)} & \cdots & W_{i, k}^{(Q-1)}\end{array}\right]$. Then, stacking up the $N_{T}$ received signal vectors results in the signal model for the NSD as

$$
\mathbf{R}_{k}=\mathbf{X}_{k} \mathbf{H}_{k}+\mathbf{W}_{k},
$$

where $\mathbf{R}_{k}=\left[\mathbf{r}_{0, k}^{T} \mathbf{r}_{1, k}^{T} \cdots \mathbf{r}_{N_{T}-1, k}^{T}\right]^{T}, \mathbf{X}_{k}=\operatorname{diag}\left\{X_{0, k}\right.$, $\left.X_{1, k}, \cdots, X_{N_{T}-1, k}\right\}, \mathbf{H}_{k}=\left[\mathbf{h}_{0, k}^{T} \mathbf{h}_{1, k}^{T} \cdots \mathbf{h}_{N_{T}-1, k}^{T}\right]^{T}$, and $\mathbf{W}_{k}=\left[\begin{array}{llll}\mathbf{w}_{0, k}^{T} & \mathbf{w}_{1, k}^{T} & \cdots & \mathbf{w}_{N_{T}-1, k}^{T}\end{array}\right]^{T}$. Then, from (4), the ML estimate of the $\left(N_{T}-1\right) \times 1$ information symbol vector $\mathbf{v}_{k}=\left[\begin{array}{llll}V_{1, k} & V_{2, k} & \cdots & V_{N_{T}-1, k}\end{array}\right]^{T}$ is given by

$$
\hat{\mathbf{v}}_{k}=\underset{\mathbf{v}_{k}}{\arg \min }\left\{\operatorname{tr}\left[\mathbf{R}_{k}^{H} \mathbf{X}_{k}\left(\boldsymbol{\Phi}^{(\mathrm{TD})}\right)^{-1} \mathbf{X}_{k}^{H} \mathbf{R}_{k}\right]\right\},
$$

where

$\boldsymbol{\Phi}^{(\mathrm{TD})}=\mathbf{C}_{\mathbf{h}}^{(\mathrm{TD})}+\sigma_{W}^{2} \mathbf{I}_{N_{T}}=\mathbf{C}_{\mathbf{h}}^{(\mathrm{TD})}+\left(\sigma_{C}^{2}+N_{0}\right) \mathbf{I}_{N_{T}}$,

and $\mathbf{C}_{\mathbf{h}}^{(\mathrm{TD})}$, the covariance matrix of $\mathbf{h}_{k}^{(q)}=$ $\left[H_{0, k}^{(q)} H_{1, k}^{(q)} \cdots H_{N_{T}-1, k}^{(q)}\right]^{T}$, is evaluated via (18) with $\Delta k=0$.

1) $C R$ : In light of (12), the noncoherent one-shot detector obtains the estimate for the information symbol $V_{i, k}$ according to

$$
\begin{aligned}
\hat{V}_{i, k} & =\underset{V_{i, k}}{\arg \max } \Re\left(\rho_{t}^{*} V_{i, k}^{*} \sum_{q=0}^{Q-1} R_{i, k}^{(q)} R_{i-1, k}^{(q)^{*}}\right) \\
& =\underset{V_{i, k}}{\arg \max } \Re\left(V_{i, k}^{*} \sum_{q=0}^{Q-1} R_{i, k}^{(q)} R_{i-1, k}^{(q)^{*}}\right) .
\end{aligned}
$$

The last step is due to that the fading correlation $\rho_{t}$ is realvalued. Thereupon, the BER of the TD-OFDM employing the
CR is evaluated via (14) by setting $\phi_{1}=\rho_{t}$ and $\Gamma=\sigma_{H}^{2} /\left(\sigma_{C}^{2}+\right.$ $\left.N_{0}\right)$.

2) DR: Based on (11), the DF detector performs symbol-bysymbol detection through

$$
\hat{V}_{i, k}=\underset{V_{i, k}}{\arg \min } \sum_{q=0}^{Q-1}\left|X_{i, k}^{*} R_{i, k}^{(q)}-\sum_{j=1}^{K} p_{j}^{K} \hat{X}_{i-j, k}^{*} R_{i-j, k}^{(q)}\right|^{2},
$$

where the prediction coefficients $\left\{p_{j}^{K}\right\}$ are calculated via (5), (6), and (23).

3) VR: According to (10), the reduced-state trellis-based sequence detector with $L^{U}$ states is defined as follows. Each state of the trellis in the $i$ th block interval is denoted as $\tilde{\Gamma}_{i, k}=$ $\left[X_{i-U, k} X_{i-U+1, k} \cdots X_{i-1, k}\right]$, and the corresponding branch metric is expressed as

$$
\begin{array}{r}
\Delta\left(\tilde{\Gamma}_{i, k}, V_{i, k}\right)=\sum_{q=0}^{Q-1} \mid X_{i, k}^{*} R_{i, k}^{(q)}-\sum_{j=1}^{U} p_{j}^{K} X_{i-j, k}^{*} R_{i-j, k}^{(q)} \\
-\left.\sum_{j=U+1}^{K} p_{j}^{K} \tilde{X}_{i-j, k}^{*} R_{i-j, k}^{(q)}\right|^{2},
\end{array}
$$

where the prediction coefficients $\left\{p_{j}^{K}\right\}$ are the same as the ones for the DR.

\section{B. $F D-O F D M$}

For the FD-OFDM, since the differential encoding and detection are independent and sequential for each OFDM blocks (see Fig. 1), only the detection in the $i$ th block interval is described here. The NSD performs optimum block detection for the entire $N-1$ information symbols as a whole. Piling up the $N$ received signal vectors given by (20) yields the signal model for the NSD as

$$
\mathbf{R}_{i}=\mathbf{X}_{i} \mathbf{H}_{i}+\mathbf{W}_{i},
$$

where $\mathbf{R}_{i}=\left[\mathbf{r}_{i, 0}^{T} \mathbf{r}_{i, 1}^{T} \cdots \mathbf{r}_{i, N-1}^{T}\right]^{T}, \mathbf{X}_{i}=\operatorname{diag}\left\{X_{i, 0}\right.$, $\left.X_{i, 1}, \cdots, X_{i, N-1}\right\}, \mathbf{H}_{i}=\left[\mathbf{h}_{i, 0}^{T} \mathbf{h}_{i, 1}^{T} \cdots \mathbf{h}_{i, N-1}^{T}\right]^{T}$, and $\mathbf{W}_{i}=\left[\begin{array}{llll}\mathbf{w}_{i, 0}^{T} & \mathbf{w}_{i, 1}^{T} & \cdots & \mathbf{w}_{i, N-1}^{T}\end{array}\right]^{T}$. From (4), one can obtain the ML estimate of the $(N-1) \times 1$ information symbol vector $\mathbf{v}_{i}=\left[\begin{array}{llll}V_{i, 1} & V_{i, 2} & \cdots & V_{i, N-1}\end{array}\right]^{T}$ through

$$
\hat{\mathbf{v}}_{i}=\underset{\mathbf{v}_{i}}{\arg \min }\left\{\operatorname{tr}\left[\mathbf{R}_{i}^{H} \mathbf{X}_{i}\left(\boldsymbol{\Phi}^{(\mathrm{FD})}\right)^{-1} \mathbf{X}_{i}^{H} \mathbf{R}_{i}\right]\right\},
$$

where

$$
\boldsymbol{\Phi}^{(\mathrm{FD})}=\mathbf{C}_{\mathbf{h}}^{(\mathrm{FD})}+\sigma_{W}^{2} \mathbf{I}_{N}=\mathbf{C}_{\mathbf{h}}^{(\mathrm{FD})}+\left(\sigma_{C}^{2}+N_{0}\right) \mathbf{I}_{N},
$$

and $\mathbf{C}_{\mathbf{h}}^{(\mathrm{FD})}$, the covariance matrix of $\mathbf{h}_{i}^{(q)}=$ $\left[H_{i, 0}^{(q)} H_{i, 1}^{(q)} \cdots H_{i, N-1}^{(q)}\right]^{T}$, is calculated via (18) with $\Delta i=0$.

1) $C R$ : In light of (12), the $C R$ evaluates the estimate for the information symbol $V_{i, k}$ according to

$$
\hat{V}_{i, k}=\underset{V_{i, k}}{\arg \max } \Re\left(\rho_{f}^{*} V_{i, k}^{*} \sum_{q=0}^{Q-1} R_{i, k}^{(q)} R_{i, k-1}^{(q)^{*}}\right) .
$$



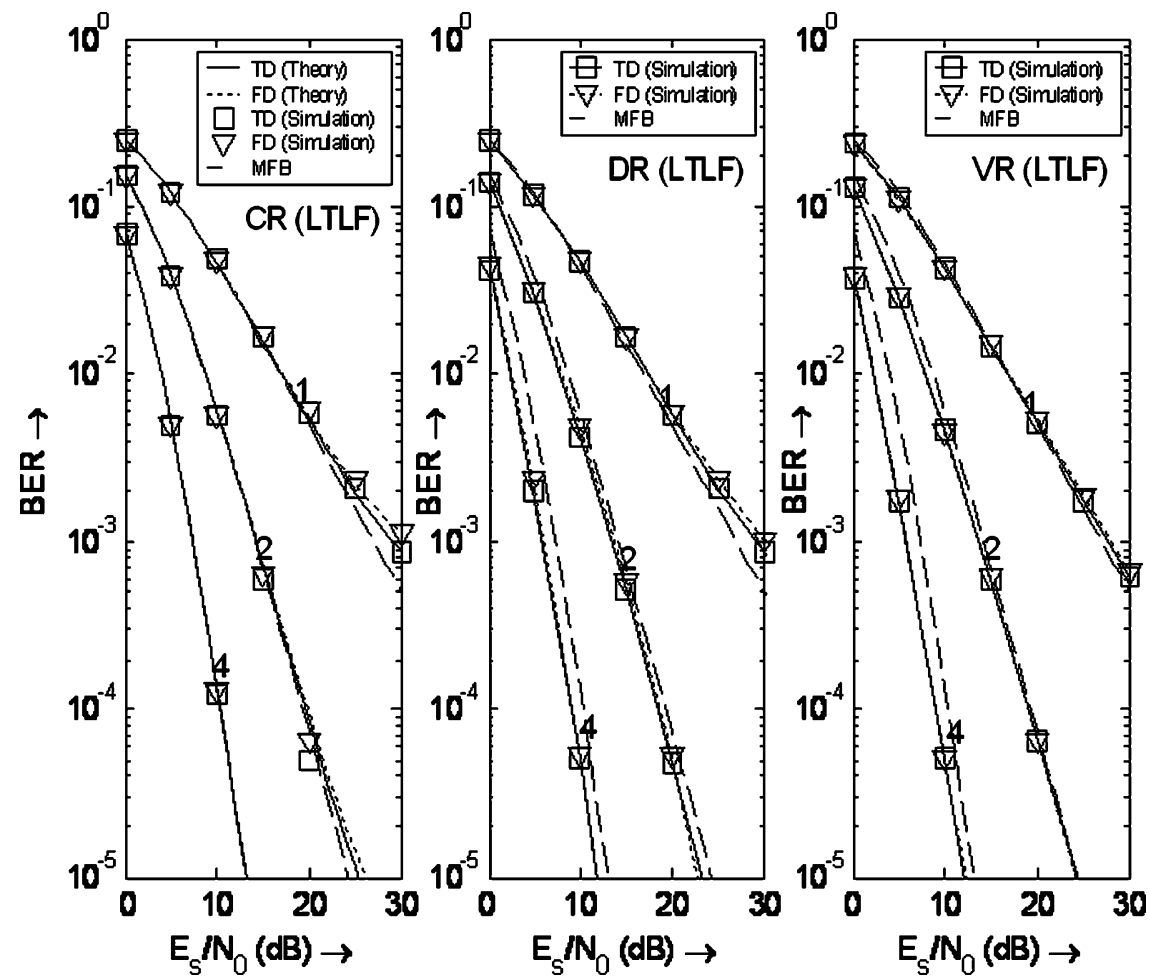

Fig. 3. BER comparisons for $\operatorname{LTLF}\left(\left|\rho_{t}\right|=0.9993 ;\left|\rho_{f}\right|=0.9988\right)$ and $Q=1,2,4$.

However, based on (13), the suboptimum detector ignores the complex-valued fading correlation $\rho_{f}$ and gets a suboptimum estimate of $V_{i, k}$ by

$$
\hat{V}_{i, k}=\underset{V_{i, k}}{\arg \max } \Re\left(V_{i, k}^{*} \sum_{q=0}^{Q-1} R_{i, k}^{(q)} R_{i, k-1}^{(q)^{*}}\right) .
$$

The BER of the FD-OFDM using this suboptimum detector is computed via (14) with the substitutions of $\phi_{1}=\rho_{f}$ and $\Gamma=$ $\sigma_{H}^{2} /\left(\sigma_{C}^{2}+N_{0}\right)$. Apparently, this BER expression is an upper bound on the performance of the FD-OFDM utilizing the CR, given by (30).

2) DR: Based on (11), the DF detector performs symbol-bysymbol detection through

$$
\hat{V}_{i, k}=\underset{V_{i, k}}{\arg \min } \sum_{q=0}^{Q-1}\left|X_{i, k}^{*} R_{i, k}^{(q)}-\sum_{j=1}^{K} p_{j}^{K} \hat{X}_{i, k-j}^{*} R_{i, k-j}^{(q)}\right|^{2},
$$

where the prediction coefficients $\left\{p_{j}^{K}\right\}$ are computed via (5), (6), and (29).

3) VR: According to (10), the reduced-state trellis-based sequence detector with $L^{U}$ states is defined as follows. Each state of the trellis for the $k$ th subcarrier is represented as $\tilde{\Gamma}_{i, k}=$ $\left[X_{i, k-U} X_{i, k-U+1} \cdots X_{i, k-1}\right]$, and the corresponding branch metric is expressed as

$$
\begin{array}{r}
\Delta\left(\tilde{\boldsymbol{\Gamma}}_{i, k}, V_{i, k}\right)=\sum_{q=0}^{Q-1} \mid X_{i, k}^{*} R_{i, k}^{(q)}-\sum_{j=1}^{U} p_{j}^{K} X_{i, k-j}^{*} R_{i, k-j}^{(q)} \\
\quad-\left.\sum_{j=U+1}^{K} p_{j}^{K} \tilde{X}_{i, k-j}^{*} R_{i, k-j}^{(q)}\right|^{2},
\end{array}
$$

where the prediction coefficients $\left\{p_{j}^{K}\right\}$ are the same as the ones for the DR.

\section{NUMERICAL RESULTS}

The simulation parameters are detailed as follows. 1) the carrier frequency and the system bandwidth are $1.8 \mathrm{GHz}$ and 800 $\mathrm{kHz}$, respectively, and thus the symbol duration is $T=1.25 \mu \mathrm{s}$; 2 ) the number of subcarriers and guard samples are $N=128$ and $G=32$, respectively, and hence the total OFDM block duration is $(N+G) T=200 \mu \mathrm{s} ; 3)$ the modulation is differential BPSK, i.e. $L=2 ; 4$ ) the number of uncorrelated paths is $M=12 ; 5$ ) the number of receive antennas is $Q=1,2$, and 4; 6) for both the DR and VR, the prediction order is $K=5$; 7) for the VR, $U=1$, i.e., the number of states is $L^{U}=2$, and the decision delay is $D=10$.

As shown in Figs. 3-6, the BER's of differential OFDM systems are compared in four extreme scenarios (see Table I), namely, LTLF, HTLF, LTHF and HTHF, respectively. For instance, LTHF stands for low time-selectivity and high frequency-selectivity. In each figure, the performances of the CR, DR, and VR are presented from left to right. The matched filter bounds (MFB's) shown in all figures are the BER's of the single-carrier differential BPSK systems employing the noncoherent one-shot detector with one-, two-, and four-branch diversity reception, respectively, in the quasistatic Rayleigh fading channel. These bounds are produced via (14) by setting $\phi_{1}=\phi_{0}=1$. Apparently, they are the lower bounds of the BER's of differential OFDM systems utilizing the CR. However, at low SNR level, they are slightly higher than the BER's of the systems using the DR or VR in all channel conditions. This is due to that the DR and VR, originated from the NSD, 

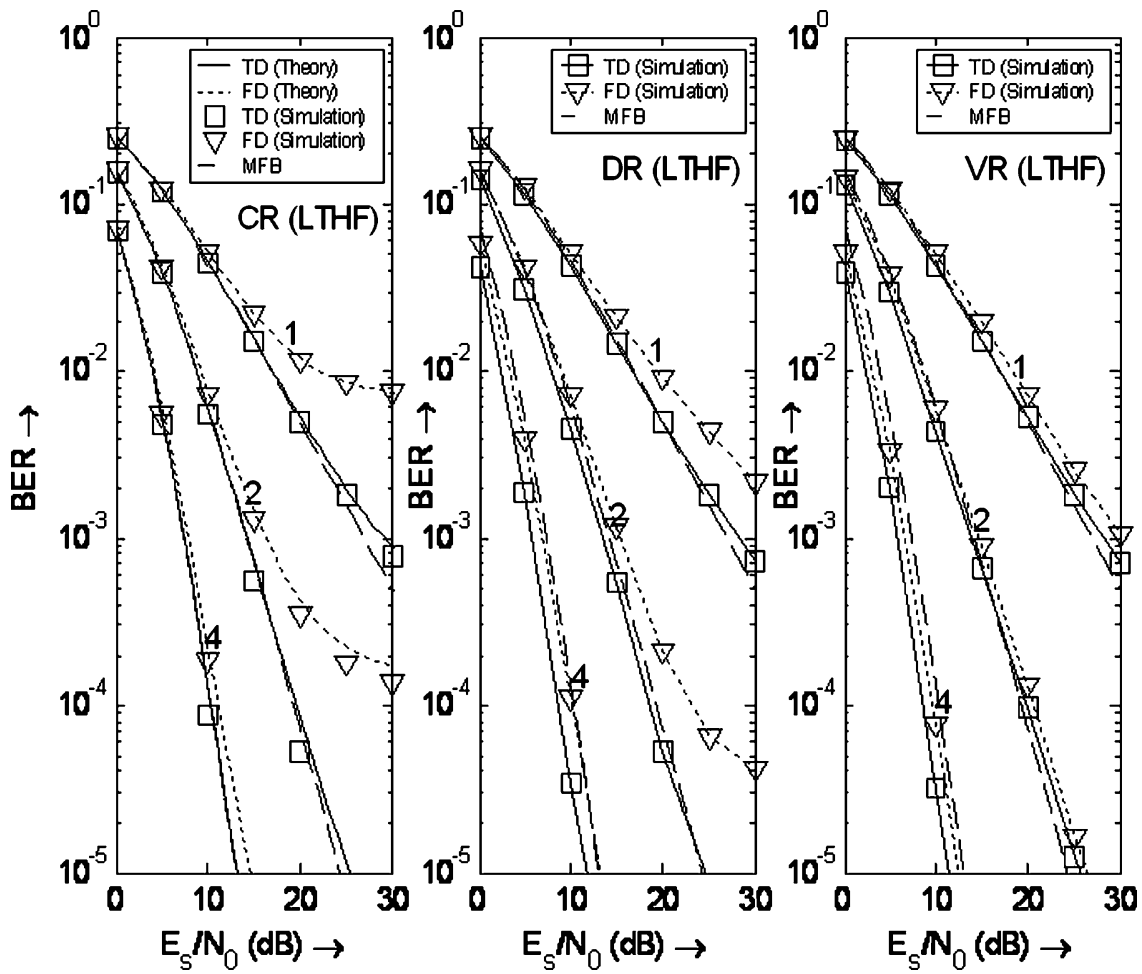

Fig. 4. BER comparisons for $\operatorname{LTHF}\left(\left|\rho_{t}\right|=0.9993 ;\left|\rho_{f}\right|=0.9859\right)$ and $Q=1,2,4$.
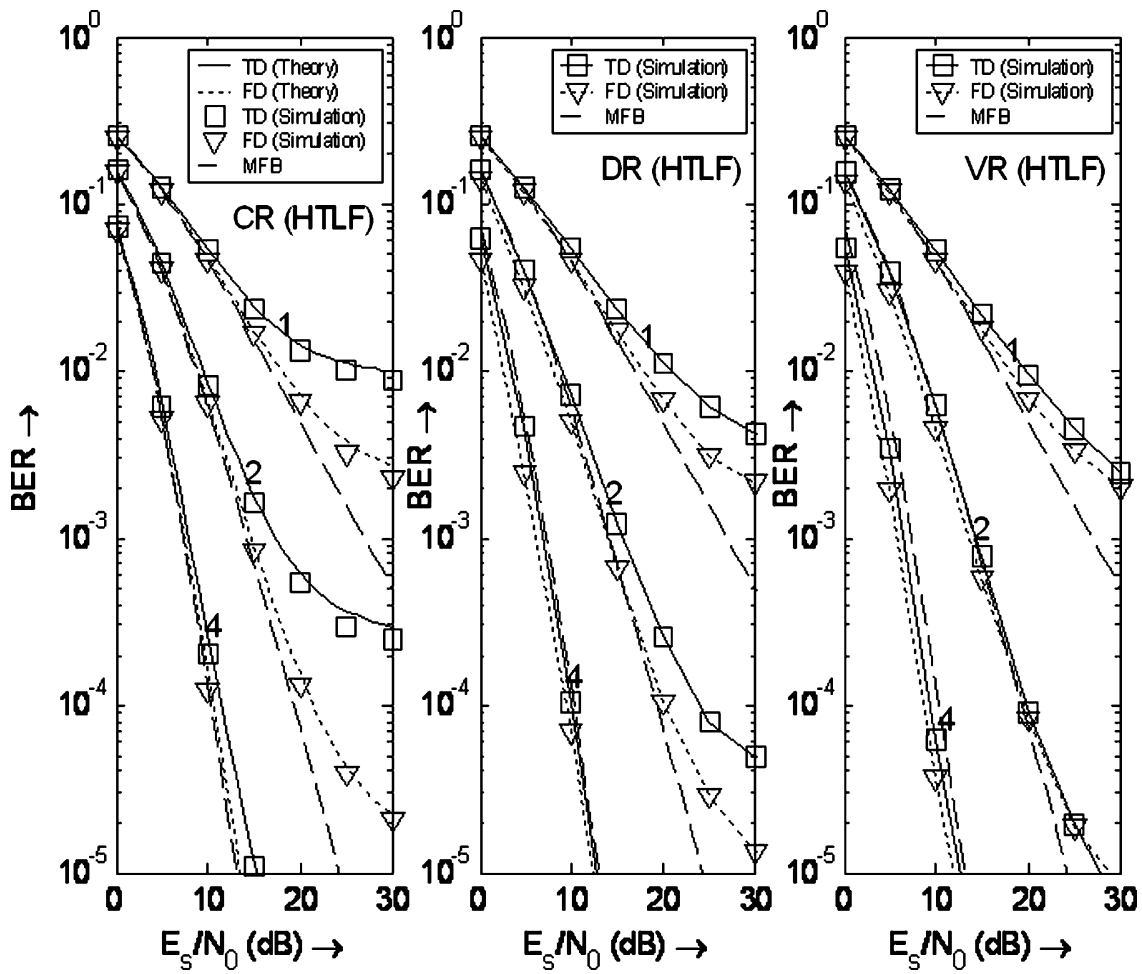

Fig. 5. BER comparisons for $\operatorname{HTLF}\left(\left|\rho_{t}\right|=0.9826 ;\left|\rho_{f}\right|=0.9972\right)$ and $Q=1,2,4$.

are inherently of better performances than the noncoherent one-shot detector (see Section II-D).

As shown in Fig. 3 (LTLF), since the channel selectivity is low, both TD-OFDM and FD-OFDM experience BER's similar to that of the corresponding MFB's regardless of which receiver structure is employed. On the other hand, as shown in Figs. 4-6, error floors appear when the channel selectivity is higher, especially for the systems utilizing the CR. The reasons for the presence of these error floors are twofold: 1) the ICI induced when the channel is not constant over an OFDM block duration; 2) the worse estimates of the fading-plus-noise processes resulted from faster channel variations along the differential detection 

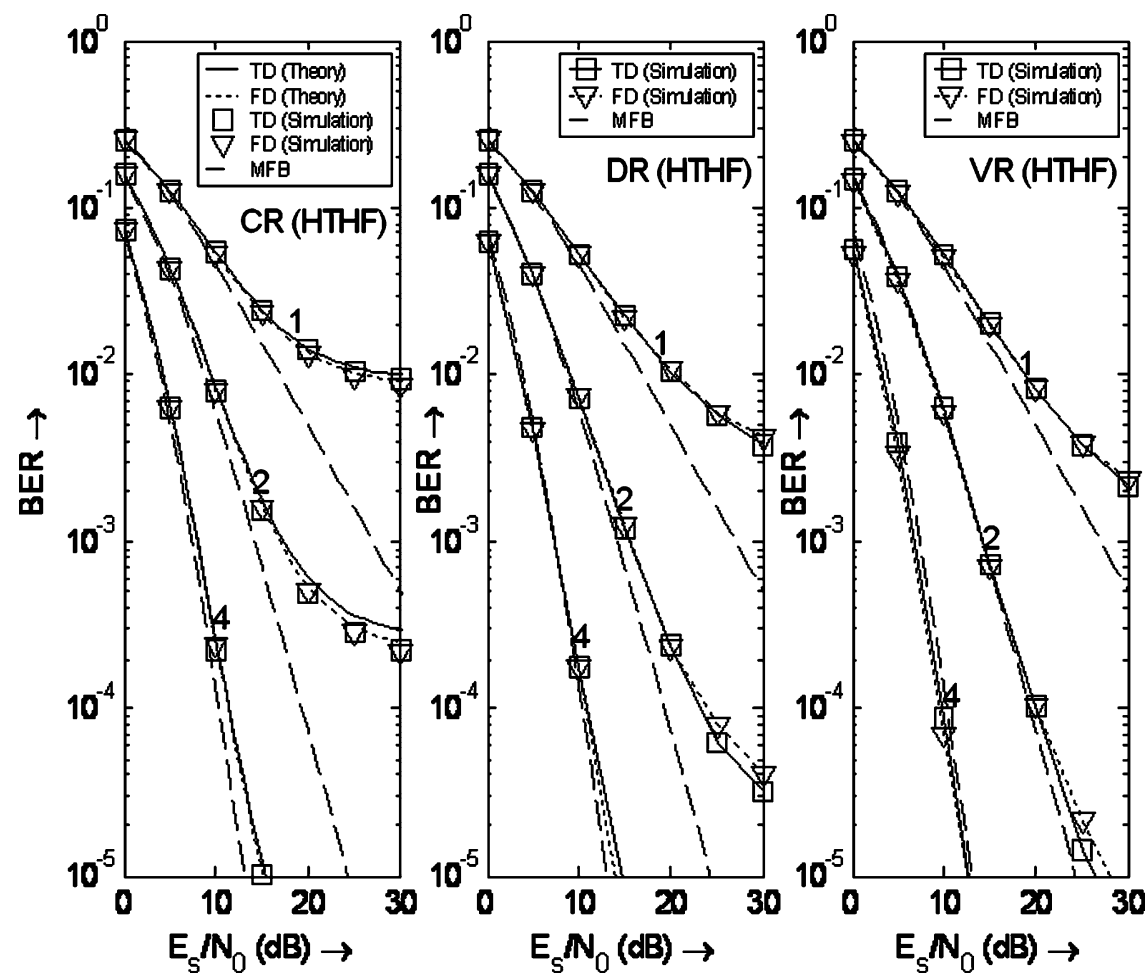

Fig. 6. BER comparisons for $\operatorname{HTHF}\left(\left|\rho_{t}\right|=0.9826 ;\left|\rho_{f}\right| 0.9843\right)$ and $Q=1,2,4$.

TABLE I

PARAMETERSETTINGS FOR DIFFERENT CHANNEL CONDITIONS

\begin{tabular}{lcc}
\hline & $\boldsymbol{f}_{D}(\mathrm{~Hz})$ & $\boldsymbol{v}(\mathrm{km} / \mathrm{hr})$ \\
\hline \hline $\mathrm{LT}$ & 40 & 24 \\
\hline $\mathrm{HT}$ & 200 & 120 \\
\hline \hline & $\tau_{\text {rms }}(\mu \mathrm{s})$ & $d$ \\
\hline \hline $\mathrm{LF}$ & 1.20 & 1 \\
\hline $\mathrm{HF}$ & 4.27 & 20 \\
\hline \hline
\end{tabular}

direction (see Section II-D). If the TD- and FD-OFDM undergo the same channel time-selectivity, the former results in the same performance degradation. However, the later lifts the error floors further due to the estimation error increases with the channel selectivity. Thereupon, they obtain similar performance for both LTLF (Fig. 3) and HTHF (Fig. 6). In addition, the TD-OFDM performs better than the FD-OFDM for LTHF (Fig. 4), whereas the TD-OFDM performs worse than the FD-OFDM for HTLF (Fig. 5).

Moreover, as observed from Fig. 5, it is evident that: 1) the VR and DR perform better than the CR; 2) the performances of the FD-OFDM with different receiver structures are similar. The former is due to that the VR and DR detect signals with the aid of better estimates (see Section II-D). The later is consequent on the low frequency-selectivity. In this case, the qualities of the estimates are good and similar. As seen from (16), the ICI is treated as an equivalent noise term, thus no reduction on the ICI is made in all receiver structures. Thereupon, the presence of these error floors is mainly resulted from the ICI.

Finally, as viewed from Fig. 6, both the VR and DR yield significant performance improvements over the $\mathrm{CR}$ when one or two receive antennas are employed. However, when four receive antennas are available, all receivers benefit from the larger spatial diversity gain and hence their performances are equally good. In this case, since the performance of the CR is satisfactory, the consideration for implementing the DR and VR is not necessary.

\section{CONCLUSIONS}

In this paper, we review the NSD and its three special cases, namely, the CR, DR, and VR. Based on the estimator-detector structures, a hierarchical interpretation of the NSD and its special cases are also presented. Then the NSD and its special cases are applied to the differential OFDM systems with diversity reception. Moreover, assuming sufficient $\mathrm{CP}$, we provide a simple closed-form BER expression for differential OFDM systems employing the $\mathrm{CR}$ with diversity reception in the time-varying multipath Rayleigh fading channels. Numerical results have revealed that, with multi-antenna diversity reception, the performance of the CR is improved significantly. However, when few receive antennas are available, the implementation of the DR or VR is necessary for achieving better and satisfactory performance.

\section{REFERENCES}

[1] H. B. Voelcker, "Phase-shift keying in fading channels," Proc. IEEE, vol. 107, pp. 31-38, Jan. 1960.

[2] P. Y. Kam, "Bit error probabilities of MDPSK over the nonselective Rayleigh fading channel with diversity reception," IEEE Trans. Commun., vol. 39, pp. 220-224, Feb. 1991.

[3] D. Divsalar and M. K. Simon, "Multiple-symbol differential detection of MPSK," IEEE Trans. Commun., vol. 38, pp. 300-308, Mar. 1990.

[4] P. Ho and D. Fung, "Error performance of multiple-symbol differential detection of PSK signals transmitted over correlated Rayleigh fading channels," IEEE Trans. Commun., vol. 40, pp. 1566-1569, Oct. 1992. 
[5] R. Schober, W. H. Gerstacker, and J. B. Huber, "Decision-feedback differential detection of MDPSK for flat Rayleigh fading channels," IEEE Trans. Commun., vol. 47, pp. 1025-1035, July 1999.

[6] X. Yu and S. Pasupathy, "Innovations-based MLSE for Rayleigh fading channels," IEEE Trans. Commun., vol. 43, pp. 1534-1544, Feb./Mar./Apr. 1995.

[7] G. M. Vitetta and D. P. Taylor, "Viterbi decoding of differentially encoded PSK signals transmitted over Rayleigh frequency-flat fading channels," IEEE Trans. Commun., vol. 43, pp. 1256-1259, Feb./Mar./Apr. 1995.

[8] R. Raheli, A. Polydoros, and C. K. Tzou, "Per-survivor processing: a general approach to MLSE in uncertain environments," IEEE Trans. Commun., vol. 43, pp. 354-364, Feb./Mar./Apr. 1995.

[9] Y. H. Kim, I. Song, H. G. Kim, T. Chang, and H. M. Kim, "Performance analysis of a coded OFDM system in time-varying multipath Rayleigh fading channels," IEEE Trans. Veh. Technol., vol. 48, pp. 1610-1615, Sep. 1999.

[10] R. V. Nee and R. Prasad, OFDM Wireless Multimedia Communications. Boston: Artech House, 2000.

[11] S. Moriyama, K. Tsuchida, and M. Sasaki, "Digital transmission of high bit rate signals using 16DAPSK-OFDM modulation scheme," IEEE Trans. Broadcast., vol. 44, pp. 115-122, Mar. 1998.

[12] V. Engles and H. Rohling, "Multi-resolution 64-DAPSK modulation in a hierarchical COFDM transmission system," IEEE Trans. Broadcast., vol. 44, pp. 139-149, Mar. 1998.
[13] M. Lott, "Comparison of frequency and time domain differential modulation in an OFDM system for wireless ATM," in Proc. IEEE Vehicular Technology Conference, vol. 2, May 1999, pp. 877-883.

[14] J. Lu, T. T. Tjhung, F. Adachi, and C. L. Huang, "BER performance of OFDM/MDPSK system in frequency-selective Ricean fading with diversity reception," IEEE Trans. Veh. Technol., vol. 49, pp. 1216-1225, July 2000.

[15] J. W. Jwa and H. S. Lee, "Performance of OFDM/MDPSK over timevariant multipath Rayleigh fading channels," IEICE Trans. Commun., vol. E84-B, pp. 337-340, Feb. 2001.

[16] "Radio Broadcasting Systems; Digital Audio Broadcasting (DAB) to Mobile, Portable and Fixed Receivers,", ETSI ETS 300 401, Feb. 1995

[17] S. Haykin, Adaptive Filter Theory, 4th ed. New Jersey: Prentice-Hall, 2002.

[18] T. K. Moon and W. C. Stirling, Mathematical Methods and Algorithms for Signal Processing. New Jersey: Prentice-Hall, 2000.

[19] D. B. Lin, P. H. Chiang, and H. J. Li, "Performance analysis of twobranch transmit diversity block coded OFDM systems in time-varying multipath Rayleigh fading channels," IEEE Trans. Veh. Technol., vol. 54, pp. 136-148, Jan. 2005.

[20] G. L. Stüber, Principles of Mobile Communication, 2nd ed. London, U.K.: Kluwer, 2001.

[21] Y. Li and L. J. Cimini Jr., "Bounds on the interchannel interference of OFDM in time-varying channels," IEEE Trans. Commun., vol. 49, pp. 401-404, Mar. 2001. 